\title{
Radiogenomics in Medulloblastoma: Can the Human Brain Compete with Artificial Intelligence and Machine Learning?
}

W e read with keen interest "MR Imaging-Based Radiomic Signatures of Distinct Molecular Subgroups of Medulloblastoma" published recently in your prestigious journal. ${ }^{1} \mathrm{We}$ would like to congratulate the authors for their multi-institutional collaborative study wherein radiomic features extracted from MR imaging coupled with machine learning led to the prediction of molecular subgroups in medulloblastoma. Recently, it has become increasing possible to extract meaningful information beyond staging from diagnostic imaging (radiophenotypes) for correlation with genomic data and molecular markers (molecular phenotypes), leading to the exciting and emerging field of radiogenomics. ${ }^{2,3}$ The introduction of computer-based algorithms (including artificial intelligence and machine learning approaches) has not only refined the assessment of semantic features but also allows simultaneous extraction of agnostic features (histograms, textures, wavelets, and fractal dimensions), which generally go beyond the limited capabilities of the human brain. Such automated high-throughput processing of big data significantly reduces time and virtually eliminates interobserver variability.

In the referenced study, Iv et al, ${ }^{1}$ extracted 590 radiomic features such as intensity-based histograms, tumor-edge sharpness, Gabor features, and local area integral invariant features from T1and T2-weighted MR imaging in a multi-institutional cohort of 109 children with medulloblastoma and used support vector machine algorithms to identify the 4 molecular subgroups: wingless (WNT), sonic hedgehog ( $\mathrm{SHH}$ ), group 3, and group 4, respectively. Receiver operating characteristic (ROC) analysis revealed better performance of the double 10-fold cross-validation model for predicting $\mathrm{SHH}$, group 3, and group 4 medulloblastoma using a combination of T1- and T2-weighted images as demonstrated by high area under the curve (AUC) values. The independent 3-dataset cross-validation strategy demonstrated acceptably high predictive accuracy of select radiomic features for $\mathrm{SHH}$ and group 4 medulloblastoma.

Most interesting, we had also reported similar results ${ }^{4}$ in a single-institution cohort of 111 patients with medulloblastoma, which, surprisingly, was not even cited by the authors. In our study, preoperative multiparametric MR imaging (primarily T1-

http://dx.doi.org/10.3174/ajnr.A6040 and T2-weighted sequences including postcontrast images) was rigorously evaluated visually by a multidisciplinary team of radiologists and clinicians (blinded to molecular subgrouping) to document and categorize a set of 13 predefined semantic imaging features and assign a probable molecular subgroup. Two-thirds of patients were chosen randomly from individual subgroups to form the training cohort, while the remaining one-third constituted the validation cohort. Multinomial logistic regression analysis was performed in the training cohort to identify imaging features with the highest discrimination of 1 subgroup from the other 3 subgroups to construct subgroup-specific binary nomograms, which were subsequently tested in the validation cohort. Predictive accuracy of subgroup-specific nomograms was defined by AUC on ROC analysis, which was highest for the SHH subgroup followed by group 4 medulloblastoma. Group 3 and WNT subgroup nomograms had suboptimal and unacceptable accuracy.

A comparison of the predictive accuracy of MR imaging features (radiomic versus semantic) for molecular subgrouping of medulloblastoma is presented in the Table. A somewhat similar (if not better) performance of the human brain compared with computer-based algorithms via artificial intelligence and machine learning approaches, though somewhat intriguing, is quite reassuring and encouraging. Neither radiomic nor semantic MR imaging features identified the WNT pathway (best survival) and group 3 medulloblastoma (worst survival) robustly and accurately, posing considerable challenges in adopting imaging biomarkers in contemporary neuro-oncologic practice. However, we do believe that the inclusion of advanced and additional MR imaging sequences like diffusion-weighted imaging, gradient-echo,

\begin{tabular}{|c|c|c|c|c|}
\hline \multirow[b]{2}{*}{$\begin{array}{c}\text { Molecular } \\
\text { Subgrouping of } \\
\text { Medulloblastoma }\end{array}$} & \multicolumn{2}{|c|}{$\begin{array}{l}\text { AUC Using } \\
\text { Radiomic MRI } \\
\text { Signatures }\end{array}$} & \multicolumn{2}{|c|}{$\begin{array}{l}\text { AUC Using } \\
\text { Semantic MRI } \\
\text { Features }\end{array}$} \\
\hline & $\begin{array}{c}\text { Double 10-Fold } \\
\text { Cross- } \\
\text { Validation } \\
\text { Model }\end{array}$ & $\begin{array}{l}\text { 3-Dataset } \\
\text { Cross- } \\
\text { Validation } \\
\text { Model }\end{array}$ & $\begin{array}{l}\text { Training } \\
\text { Cohort } \\
(n=76)\end{array}$ & $\begin{array}{c}\text { Validation } \\
\text { Cohort } \\
(n=35)\end{array}$ \\
\hline WNT & 0.45 & 0.45 & 0.75 & 0.69 \\
\hline $\mathrm{SHH}$ & 0.79 & 0.70 & 0.94 & 0.99 \\
\hline Group 3 & 0.70 & 0.39 & 0.73 & 0.60 \\
\hline Group 4 & 0.83 & 0.80 & 0.85 & 0.79 \\
\hline
\end{tabular}


and spectroscopy, coupled with further technologic refinement of advanced machine-classifier tools could potentially further improve the predictive accuracy of such radiogenomic correlation.

\section{REFERENCES}

1. Iv M, Zhou M, Shpanskaya K, et al. MR imaging-based radiomic signatures of distinct molecular subgroups of medulloblastoma. AJNR Am J Neuroradiol 2019;40:154-61 CrossRef Medline

2. Kuo MD, Jamshidi N. Behind the numbers: decoding molecular phenotypes with radiogenomics: guiding principles and technical considerations. Radiology 2014;270:320-25 CrossRef Medline

3. Zhou M, Scott J, Chaudhury B, et al. Radiomics in brain tumor: image assessment, quantitative feature descriptors, and machinelearning approaches. AJNR Am J Neuroradiol 2018;39:208-16 CrossRef Medline

4. Dasgupta A, Gupta T, Pungavkar S, et al. Nomograms based on preoperative multiparametric magnetic resonance imaging for prediction of molecular subgrouping in medulloblastoma: results from a radiogenomics study of 111 patients. Neuro Oncol 2019;21:115-24 CrossRef Medline

(1) A. Dasgupta (D)T. Gupta Department of Radiation Oncology Tata Memorial Centre Mumbai, India 